\title{
CHARACTERIZATION AND IMAGING WITH LAMB WAVE LENS AT GIGAHERTZ FREQUENCIES
}

\author{
A. Bozkurt, G. Yaralığlu, A. Atalar, H. Köymen, K. Krämer † \\ Electrical and Electronics Engineering Department \\ Bilkent University, Ankara, TURKEY 06533 \\ $\dagger$ Krämer Scientific Instruments, Herborn, GERMANY 35745
}

\begin{abstract}
Lamb wave lenses with conical refracting surfaces are fabricated for use at $400 \mathrm{MHz}$ and $1 \mathrm{GHz}$. The conical surfaces are ground and polished with mechanical means and they are sufficiently smooth for the frequencies of interest. The wide bandwidth of transducers allow frequency tuning necessary for Lamb wave lenses. The fabricated lenses show the expected $V(Z)$ performance. At high frequencies the attenuation in the coupling medium can be very high, but due to the smaller wavelength the resolution is better and defocus distance can be reduced. Inherently higher leaky wave sensitivity of Lamb wave lens enables a good $V(Z)$ characterization ability at higher frequencies as compared to the conventional spherical lens.

Subsurface imaging with these Lamb wave lenses gives satisfactory results for layered structures. Chosen object has leaky wave modes within the angular coverage of the lens. The images exhibit a resolution close to the diffraction limit.

Experimental $V(Z)$ curves obtained with these lenses along with images are presented.
\end{abstract}

\section{INTRODUCTION}

The Lamb wave lens (LWL) [1] was proposed for imaging and characterization of layered structures. It differs from a conventional acoustic microscope lens in that it has a conical refracting element, which is able to selectively excite surface wave modes. This implies a higher subsurface wave excitation efficiency compared to the spherical lens. The LWL images obtained are easy to interpret since only one mode is involved. Due to higher efficiency of leaky wave excitation, a higher accuracy in characterization is expected. Previously we have demonstrated the LWL at relatively low frequencies. In this paper we report LWLs operating at $400 \mathrm{MHz}$ and $1 \mathrm{GHz}$. We present images obtained with this lens.

\section{LENS GEOMETRY}

LWLs have been designed to operate at $400 \mathrm{MHz}$ and $1 \mathrm{GHz}$ with the dimensions depicted in Fig. 1. The

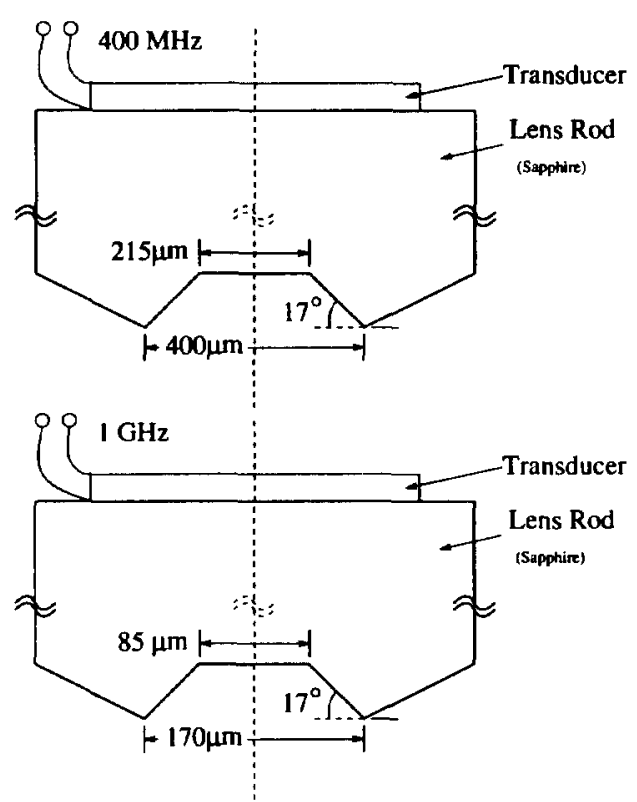

Figure 1: Dimensions of the Lamb wave lenses

lenses are fabricated on sapphire rods with a transducer geometry very similar to the conventional lens. The conical LWL cavities are ground on the opposite end of sapplise rods using mechanical means. An optical microscope image of the lens is given in Fig. 2. The lens surface is suficiently smooth and conical surfaces are flat within one wavelength. The lens surfaces are coated with a quarter wavelength glass layers to act as matching medium between sapphire and water. The associated angular spectrum at the front focal plane is in Fig. 3. The cone angle of the LWLs are chosen to be small one suitable to use with high velocity materials such as sapphire, alumina and MgO. These materials are particularly difficult to penetrate with conventional lenses due to their high impedances and narrow angular width for surface wave excitation. In particular, for $\mathrm{MgO}, V_{l}=8852 \mathrm{~m} / \mathrm{sec}$ and $V_{l}=6368 \mathrm{~m} / \mathrm{sec}$ [2]. LWL is suitable for penetration into high impedance materials 


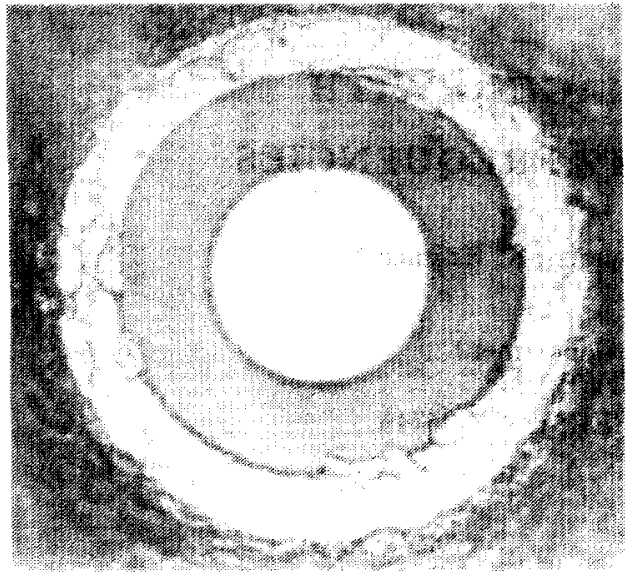

Figure 2: Optical micrograph of Lamb wave lens operating at $400 \mathrm{MHz}$

with good efficiency [3]. The calculated reflection coefficients [4] for sapphire, $\mathrm{MgO}$ and alumina are provided in Fig. 4. The phase transitions for these materials coincide with the peak in the angular spectrum of the LWL, indicating a proper combination. This particular lens can be used for materials with leaky wave velocities in the range 5100 to $6100 \mathrm{~m} / \mathrm{sec}$.

Overlapping and hence interfering specular and leaky wave signals are desirable for characterization purposes, such a combination results in periodic variations in the $V(Z)$ curve. To achieve this RF-pulse width must be sufficiently long. On the other hand, when the LWL is used for imaging purposes, poor resolution of the unfocused central beam is a problem. To improve resolution, the effect of the central beam can be eliminated by using sufficiently short pulses and time gating. It is possible to choose only the leaky wave echo amplitude for image generation. When the defocus distance is sufficient, the specular and leaky parts can be separated in time.

\section{EXPERIMENTS}

We have performed experiments with the $400 \mathrm{MHz}$ LWL. In Fig. 5, two oscilloscope traces are provided for the lens operating at $380 \mathrm{MHz}$. Referring to Fig. 6, the first pulse is the spurious signal generated from the front surface of the lens. The second pulse is the specular central beam echo and the third pulse, if exists, is the leaky wave echo. In the first oscilloscope trace, the specular and leaky wave echoes can be seen well separated using a pulse width of $25 \mathrm{nsec}$. The second trace depicts the return signal for a material on which no leaky wave modes can be excited. About 30 nsec separation between the pulses is consistent with the path differences of the rays depicted in Fig. 6. Fig. 7 depicts experimentally measured amplitude variations of specular and leaky wave echos as a function of parameter

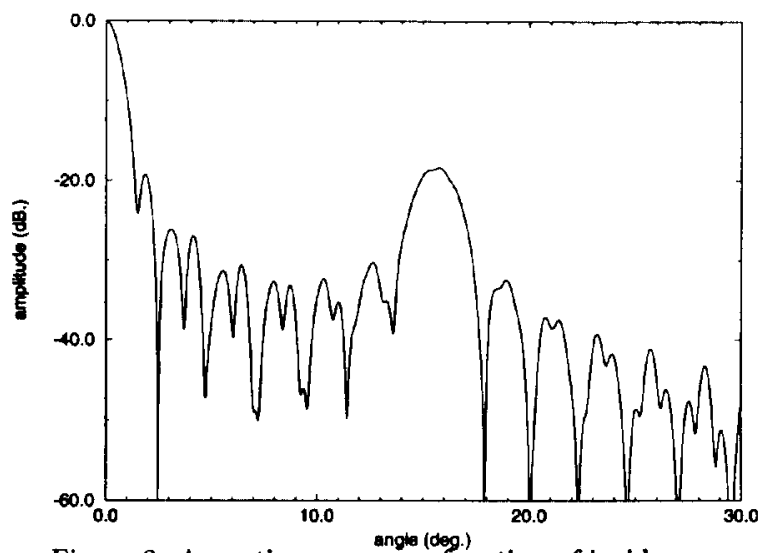

Figure 3: Acoustic power as a function of incidence angle.

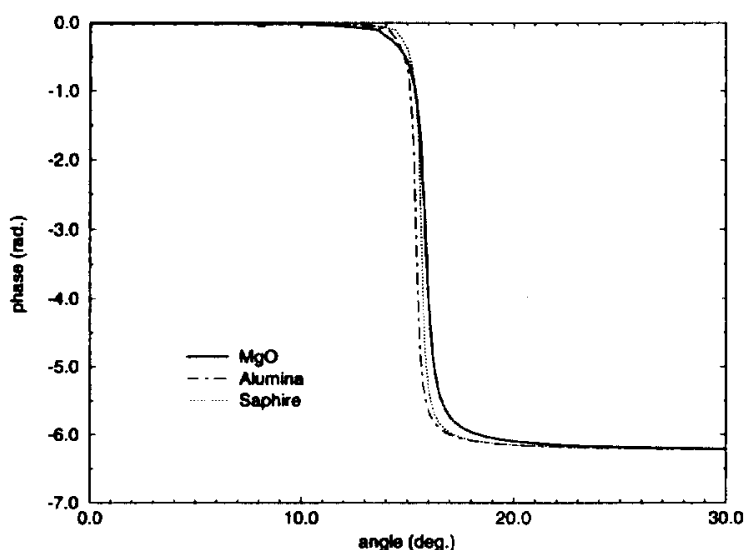

Figure 4: Calculated phase of reflection coefficients for $\mathrm{MgO}$, alumina and sapphire at the water interface. Piezoelectricity is neglected.

$z$. These curves are also in agreement with expectations [5]. The leaky wave part, $V_{L}(z)$, increases as $z$ gets close to zero, i.c., when the subsurface propagation distance is minimum. The specular or geometric part, $V_{G}(z)$, on the other hand, grows when the object lens spacing is reduced. Fig. 8 is a calculated plot of the same quantities. These curves are obtained using a simulation program which uses ray theory arguments to include the effect of the refracting element and angular spectrum approach elsewhere to take care of diffraction. To obtain the $V_{L}(z)$ curve, no central beam is assumed to originate from the lens, which is, in effect, equivalent to blocking the center. Similarly, to get the $V_{G}(z)$ curve, the conical beam is suppressed. The agreement between the theoretical and experimental $V_{G}(z)$ curves is excellent. However, a good match between the $V_{L}(z)$ curves can be achieved only when the surface wave attenuation in the object material is included.

For imaging purposes, a high frequency measurement electronics has been integrated with a commer- 
Acquisition 15 complete
$4.00050 / 3$

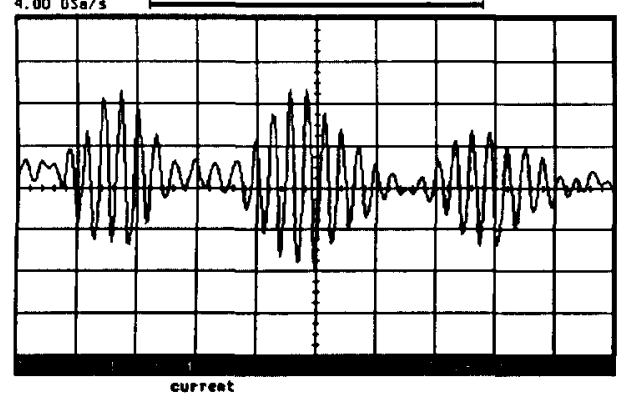

$v \operatorname{cox}(1) \quad$ current

Aequi sition is stopped

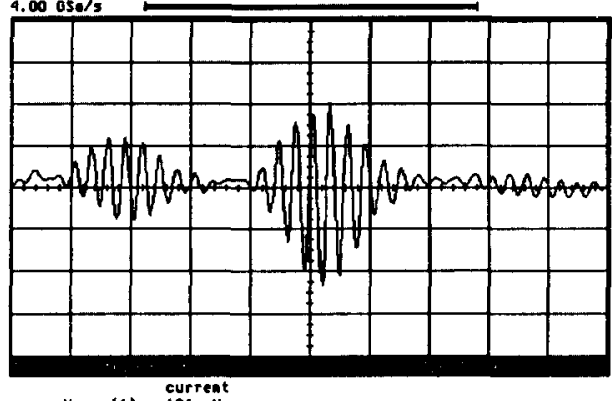

$4 \max (1)$ current

Figure 5: Two oscilloscope traces of reflected signals from a $380 \mathrm{MHz}$ Lamb wave lens for two different objects. The leaky wave echo can be seen in the first one since the cone angle matches the critical angle while for the second image no leaky wave contribution exists and hence the third pulse is not visible.

cial acoustic microscope ${ }^{1}$ which normally operates below $100 \mathrm{MHz}$. The high frequency electronics provides synthesizer generated tone bursts where the synthesizer is synchronized with the pulse repetition rate. The commercial acoustic microscope controls the mechanical $x-y$ scan system, provides the pulse repetition rate and is able to read the detected signal level in digital form. Data are interpreted by a computer, which provides a easy-to-use interface for viewing, image format conversion and archiving purposes. The system enables the imaging with a minimum pixel spacing of $4 \mu \mathrm{m}$.

There are two alignment requirements on the scanner-lens system for images of satisfactory quality. First of all, the lens axis should be perpendicular to the specimen surface for maximum signal reception. However, this adjustment does not need not to be perfect while the conical beam has a finite angular width. There will be negligible reduction in the leaky signal echo with a slight misalignment. Secondly, the scan plane needs to be aligned with the sample. This assures that the lens-specimen spacing remains unaltered as the lens is moved back and forth during scanning. IIence, the re-

\footnotetext{
${ }^{1}$ Krämer Scientific Instruments SAM 100 Acoustic Microscope
}

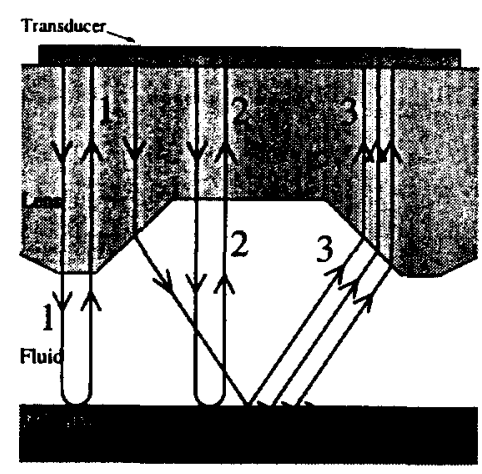

Figure 6: Rays indicating different pulses seen on the oscilloscope traces.

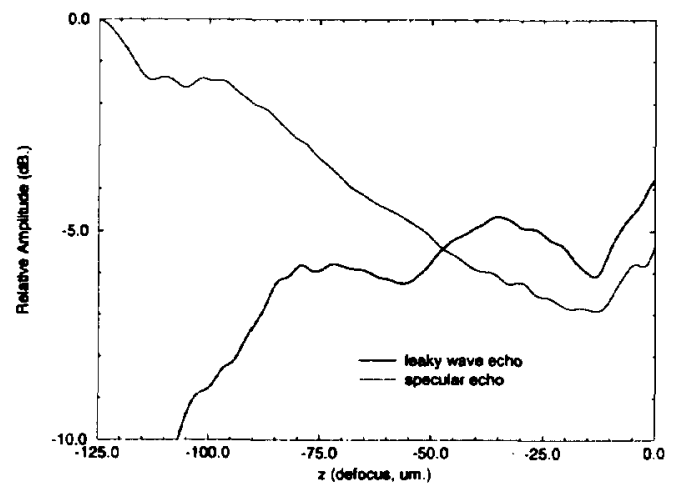

Figure 7: Measured amplitude variations of specular and leaky wave echoes as a function of distance between the lens and the $\mathrm{MgO}$ surface. Operating frequency is $380 \mathrm{MHz}$.

turn signals stay within a certain time interval, enabling time gating for measurement purposes.

Fig. 9 and Fig. 10 are acoustic images of unpolished surface of a $\mathrm{MgO}$ crystal sample obtained with this syslem at $380 \mathrm{MIIz}$. The first image uses the specular echo for comparison purposes while the second uses the leaky wave echo. The first image has a poor resolution due to the unfocused nature of the central beam. Image shows a resolution of about $150 \mu \mathrm{m}$. Since the flat portion at the center of the lens has a diameter of $200 \mu \mathrm{m}$, the resolution is in that order. Obviously, the second image has a better resolution. The wavelength of leaky wave at the operation frequency is about $16 \mu \mathrm{m}$, and the expected resolution is in the same order [6]. The observed subsurface resolution on the image is about $30 \mu \mathrm{m}$. Since $\mathrm{MgO}$ is a high velocity material, resolution is poorer compared to that in lower velocity materials.

If the object under investigation has layers, frequency tuning may be necessary to match a leaky mode to the fixed incidence angle of the lens. 


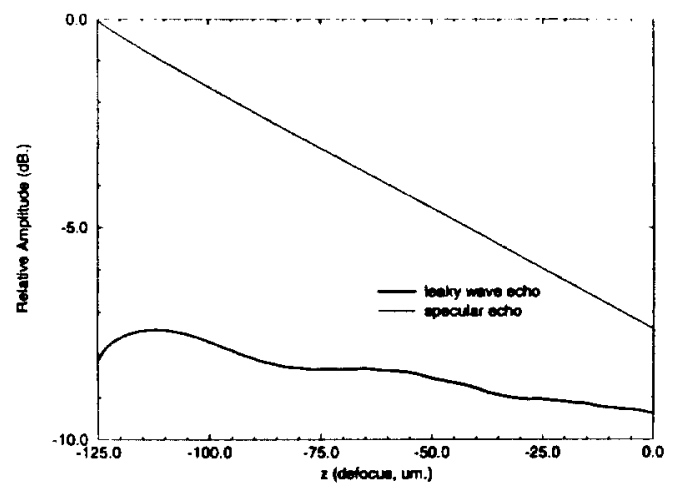

Figure 8: Calculated amplitude variations of specular and leaky wave echoes as a function of distance between the lens and the $\mathrm{MgO}$ surface. The surface wave attenuation in $\mathrm{MgO}$ is neglected. Operating frequency is $380 \mathrm{MHz}$.

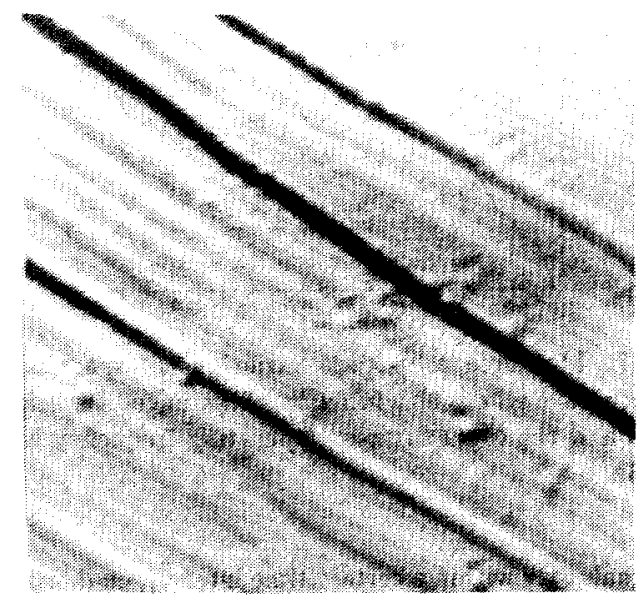

Figure 9: Image of $\mathrm{MgO}$ surface using specular echo. Image dimensions are $3 \mathrm{~mm}$ by $3 \mathrm{~mm}$.

\section{CONCLUSION}

We have demonstrated the feasibility of manufacturing Lamb wave lenses at high frequencies. The Lamb wave lens is adapted for use in a commercial acoustic microscope. We have presented subsurface images of high velocity materials which are hard to penetrate. If sufficiently short RF-pulses are used it is possible to separate leaky wave and specular echos in the time domain, and there is no need to block the central beam for imaging purposes. The separation of pulses also permits the determination of $V_{L}(z)$ and $V_{G}(z)$ curves directly. It is also shown that resolution obtained using leaky waves can be close to the theoretical limit.

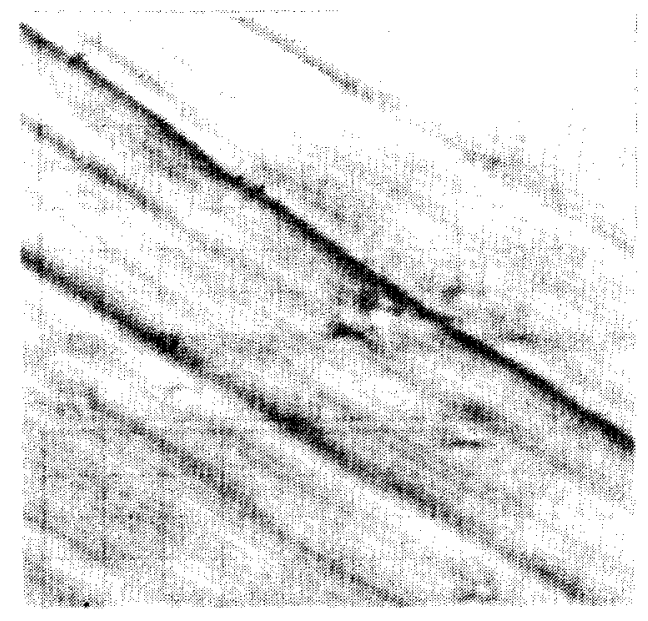

Figure 10: Image of $\mathrm{MgO}$ surface using the leaky wave echo. Image dimensions are $3 \mathrm{~mm}$ by $3 \mathrm{~mm}$.

\section{References}

(1] A. Atalar and H. Köymen "A high efficiency Lamb wave lens for subsurface imaging," in Proc. of IEEE 1989 Ultmasonics Symposium, pp. 813-816, 1989.

[2] A. Briggs. Acoustic Microscopy. Oxford University Press, Oxford, 1992.

[3] A. Atalar, L. Değertekin, and H. Köymen "Recent Advances in Acoustic Microscopy," in IEEE Ultrasonic Proceedings, pp. 719-726, 1991.

[4] L.M. Brekhovskikh. Waves in Layered Media. Academic Press, New York, 1980.

[5] II.L. Bertoni "Ray-optical evaluation of $V(Z)$ in the reflection acoustic microscope," IEEE Trans. Son. Ultrason., vol. 31, pp. 105-116, 1984.

[6] N. Günalp and A. Atalar "Response of acoustic imaging systems using convergent leaky waves to cylindrical flaws," IEEE Trans. on Ultrason. Ferra. and Freq. Cont., vol. 36, pp. 507-516, 1989. 